\title{
RESEARCH \\ High-resolution magnetic resonance imaging and diffusion tensor imaging of the porcine temporomandibular joint disc
}

\author{
E Benavides*,1, M Bilgen ${ }^{2}$, B Al-Hafez ${ }^{3}$, T Alrefae $^{4}$, Y Wang ${ }^{5}$ and P Spencer ${ }^{6,7}$ \\ ${ }^{I}$ Department of Periodontics and Oral Medicine, University of Michigan, Michigan, USA; ${ }^{2}$ Department of Radiology, Medical \\ University of South Carolina, South Carolina, USA; ${ }^{3}$ Department of Surgery, University of Arkansas for Medical Sciences, \\ Arkansas, USA; ${ }^{4}$ Department of Physics, Kuwait University, Kuwait; ${ }^{5}$ Department of Oral Biology University of Missouri Kansas \\ City, Missouri, USA; ${ }^{6}$ Bioengineering Research Center, University of Kansas, Kansas, USA; ${ }^{7}$ Department of Mechanical \\ Engineering, University of Kansas, Kansas, USA
}

Objectives: Diffusion tensor imaging (DTI) is an MRI modality for characterizing the property, microstructural organization and function in tissues such as the brain and spinal cord. Prior to this investigation, DTI had not been adapted for studies of the temporomandibular joint (TMJ) disc.

Objectives were to test the feasibility of DTI to evaluate the porcine TMJ disc and to use DTI to observe differences in magnitude of anisotropy of water diffusion between TMJ disc regions.

Methods: Five adult pig TMJs were scanned on a 9.4 Tesla horizontal bore MRI scanner using an inductively coupled surface coil. High-resolution gradient-echo and diffusionweighted spin-echo based images were obtained. The mean diffusivity and fractional anisotropy (FA) were computed in different regions of the disc. Two observers were calibrated to review the two-dimensional and three-dimensional images. Polarized light microscopy was used as the gold standard for collagen fibre orientation.

Results: In the sagittal plane, the mean diffusivity was higher in the posterior $\left(1.28 \pm 0.10 \times 10^{-3} \mathrm{~mm}^{-2} \mathrm{~s}^{-1}\right)$ and anterior $\left(1.27 \pm 0.08 \times 10^{-3} \mathrm{~mm}^{-2} \mathrm{~s}^{-1}\right)$ bands compared with the intermediate zone $\left(0.96 \pm 0.01 \times 10^{-3} \mathrm{~mm}^{-2} \mathrm{~s}^{-1}\right)$, and the FA index was also lowest in the intermediate zone. In the coronal plane, the mean diffusivity was higher in the medial $\left(1.42 \pm 0.01 \times 10^{-3} \mathrm{~mm}^{-2} \mathrm{~s}^{-1}\right)$ and lateral $\left(1.21 \pm 0.12 \times 10^{-3} \mathrm{~mm}^{-2} \mathrm{~s}^{-1}\right)$ aspects than in the centre $\left(1.09 \pm 0.08 \times 10^{-3} \mathrm{~mm}^{-2} \mathrm{~s}^{-1}\right)$, and the FA index was also lowest in the centre.

Conclusions: DTI is a useful method for non-invasively characterizing the structure/ property relationships of the porcine TMJ disc.

Dentomaxillofacial Radiology (2009) 38, 148-155. doi: 10.1259/dmfr/19195745

Keywords: magnetic resonance imaging, diffusion tensor imaging, surface coil, temporomandibular joint disc

\section{Introduction}

The temporomandibular joint (TMJ) disc is a dense fibrocartilaginous microheterogeneous tissue located between the two bony components of the TMJ. The disc, which serves as the principal mechanism for stress distribution and lubrication, presents distinct regional variations in its biochemistry, microstructure and

*Correspondence to: Dr Erika Benavides, Department of Periodontics and Oral Medicine, University of Michigan, School of Dentistry Office 2029A, 1011 North University Avenue, Ann Arbor, MI 48109-1078, USA; E-mail: benavid@umich.edu

Received 2 November 2007; revised 2 April 2008; accepted 3 April 2008 biomechanics. $^{1-4}$ This variation is related in part to regional variation in the collagen fibre orientation. ${ }^{4-7}$ Specifically, in the posterior and anterior bands of the disc, the collagen fibres run in a mediolateral direction, whereas the fibres in the intermediate zone are oriented anterior to posterior. This regular pattern of organization is disrupted as a consequence of disease or displacement of the disc. ${ }^{6,8}$

Our current efforts are focused on investigating the structure, properties and function of the porcine TMJ disc at both macroscopic and microscopic scales. As part of our studies we have tested the potential of 
applying complex information-rich MRI modalities to probe the disc. MRI is currently considered to be the best anatomical imaging technique for non-invasively evaluating the soft tissues of the TMJ. ${ }^{9-13}$ The porcine disc has found common acceptance in TMD research because it mimics the anatomy and function seen in humans. ${ }^{14}$ The porcine TMJ has been examined earlier using high-resolution MRI; however, these previous studies are limited in number and only provide morphological information restricted to the appearance of the condylar cartilage on anatomical MRI. ${ }^{15,16}$

Diffusion tensor imaging (DTI) is an MRI modality that has the potential for detecting, measuring and quantifying microstructural changes in organized and semi-organized tissues as well as for providing threedimensional (3D) information about the microstructural organization of fibres and structures in a noninvasive manner. DTI has been applied in several studies to infer the microstructural characteristics of the brain, the heart and the spinal cord. ${ }^{17-20}$ In the brain and spinal cord it has been used for nerve fibre tracking, which has proven to be clinically successful for the detection of early pathology on these tissues.

In addition, DTI has been introduced in the clinical diagnosis of disease conditions such as cerebral ischaemia, acute stroke and multiple sclerosis. ${ }^{21-23}$ Unlike conventional diffusion-weighted imaging (DWI), where diffusion-weighted (DW) images are used to calculate the scalar apparent diffusion coefficient (ADC), DTI characterizes diffusive transport of water by an effective diffusion tensor $D .{ }^{24}$ The eigenvalues of $D$ are the three principal diffusivities and the eigenvectors define the local fibre tract direction field. ${ }^{25}$ Moreover, one can derive from $D$ rotationally invariant scalar quantities that describe the intrinsic diffusion properties of the tissue. The most commonly used are the trace of the tensor, which measures mean diffusivity, fractional anisotropy (FA) and lattice index (LI), which characterize the anisotropy of the fibre structure, meaning how much higher the diffusivity is along some directions compared with others. ${ }^{25-28}$

The usefulness of DTI for the detection of microstructural changes that are indicative of differences in the micromechanical properties of the TMJ disc had not been explored prior to this project. In this article, we present the results from the first adaptation of DTI to an investigation of the TMJ disc. DTI is a relatively recent MRI modality for relating image intensities to relative mobility of endogenous tissue water molecules and was used to study the degree of anisotropy in which the water molecules move across the tissue, which would be indicative of the orientation and organization of the collagen fibres. As described above, it is a well accepted imaging modality in clinical and experimental radiology to evaluate the orientation, distribution, size and integrity of myelinated axonal neuronal fibres. DTI was used in this study to map the orientation of the collagen fibres and to characterize the diffusion proper- ties of the TMJ disc. We hypothesize that DTI can provide information on the intact TMJ disc that is currently unavailable with other non-invasive imaging techniques. Here, we test this hypothesis with experimental studies on the porcine TMJ disc and determine the validity of DTI to characterize the structure/ property relationships of the intact TMJ disc.

\section{Materials and methods}

Five excised frozen heads from female Yorkshire $x$ Landrace cross adult pigs, ages 12-16 months, were obtained from the Swine Resource Center at the University of Missouri-Columbia. The animals were utilized in cardiovascular studies previously approved by the appropriate Animal Use Committee. The acquisition of pig heads for this work was considered incidental to other work and thus the protocol was exempt. Using an autopsy saw, ten joints were harvested. One joint per animal was randomly selected for a total of five joints to be evaluated using DTI. The other five joints were designated for another project. The temporal bone was carefully removed by separating the joint capsule from the articular surface of the bone while leaving the intact discs attached to the condyle. The discs appeared normal and healthy upon gross examination. The samples were tightly wrapped in plastic to prevent dehydration.

The TMJ samples were scanned at room temperature on a 9.4 Tesla horizontal bore scanner (Varian Inc., Palo Alto, CA), using an inductively coupled surface coil positioned in contact with the superior aspect of the disc along an axis parallel to the mediolateral direction. ${ }^{29}$ The specimen-coil assembly was placed on a Plexiglas ${ }^{\circledR}$ cradle and introduced into the magnet for imaging. After confirming the assembly position in the magnet's isocentre, high-resolution multislice interleaved images were acquired in axial, sagittal and coronal planes using a 3D gradient-echo (GE) sequence with the following parameters: repetition time (TR)/echo time $(\mathrm{TE})=45$ / $4.46 \mathrm{~ms}$, flip angle $=45^{\circ}$, applied over a volume of $31 \times 20 \times 14 \mathrm{~mm}^{3}$, where the former represents the readout direction, image matrix $=256 \times 128 \times 128$ and number of excitations $=2$. With these settings, the spatial resolution was $70-100 \mu \mathrm{m}$. Diffusion-weighted images were obtained with a spin-echo-based sequence. The diffusion gradient pulses were set at strength of $8 \mathrm{G} \mathrm{cm}^{-1}$, duration (width, $\delta$ ) of $6.5 \mathrm{~ms}$ and a time interval (separation, $\Delta$ between the onset of the pulses) of $11 \mathrm{~ms}$ to produce a $b$-value of $346 \mathrm{~s} \mathrm{~mm}^{-2}$. After a baseline acquisition with no diffusion-sensitizing gradient $(0,0,0)$, six more measurements were obtained by applying diffusion gradients in six isotropically distributed directions $(110,101,011,-110,-101,0-11)$. The other parameters were $\mathrm{TR} / \mathrm{TE}=2000 / 24 \mathrm{~ms}$, FOV $=10 \times 10 \mathrm{~mm}^{2}$, image matrix $=128 \times 128$ (corresponding to spatial resolution of $78 \times 78 \mu \mathrm{m}^{2}$ ), slice thickness 
$=1 \mathrm{~mm}$ and number of excitations $=2$. The acquisition time was $\sim 1 \mathrm{~h}$ per specimen.

\section{Data processing}

The acquired raw data were processed and displayed using VNMRJ.1D scanner software. The overall organization of the collagen fibres in the disc was visualized using spin-echo or GE data in two and three dimensions using ANALYZE software (AnalyzeDirect Inc., Lenexa, KS). The diffusion-weighted images were processed using DTI analysis package developed under MATLAB ${ }^{\circledR}$ platform (Matworks Inc., Natick, MA). A MATLAB code was developed to analyse the data on a pixel-by-pixel basis. The code starts by applying a Gaussian filter to reduce the noise in all seven images of the slice being analysed. After prompting the user for the $b$-value used in the experiment, the code computes the measured ADC that correspond to each of the six directions where the magnetic field gradients were applied. For example, the ADC corresponding to gradients applied in the $x$ and $y$ directions simultaneously are computed as:

$$
\mathrm{ADC}_{110}=-\frac{1}{b} \ln \left(\frac{\text { Image }_{110}}{\text { Image }_{000}}\right)
$$

where Image I10 $_{10}$ is the image obtained by applying magnetic field gradients in the $x$ and $y$ directions simultaneously, and Image $_{000}$ is the image obtained without applying magnetic field gradients. After computing all six $\mathrm{ADCs}$, namely $\mathrm{ADC}_{110}, \mathrm{ADC}_{101}$, $\mathrm{ADC}_{011}, \mathrm{ADC}_{-110}, \mathrm{ADC}_{-101}$ and $\mathrm{ADC}_{0-11}$, the code computes the diffusion tensor elements $(D)$ by utilizing the following relations

$$
\begin{aligned}
& \mathrm{ADC}_{110}=D_{x x}+D_{y y}+2 \cdot D_{x y} \\
& \mathrm{ADC}_{101}=D_{x x}+D_{z z}+2 \cdot D_{x z} \\
& \mathrm{ADC}_{011}=D_{y y}+D_{z z}+2 \cdot D_{y z} \\
& \mathrm{ADC}_{-110}=D_{x x}+D_{y y}-2 \cdot D_{x y} \\
& \mathrm{ADC}_{-101}=D_{x x}+D_{z z}-2 \cdot D_{x z} \\
& \mathrm{ADC}_{0-11}=D_{y y}+D_{z z}-2 \cdot D_{y z}
\end{aligned}
$$

This may be expressed in the matrix form

$$
\left(\begin{array}{l}
\mathrm{ADC}_{110} \\
\mathrm{ADC}_{101} \\
\mathrm{ADC}_{011} \\
\mathrm{ADC}_{-110} \\
\mathrm{ADC}_{-101} \\
\mathrm{ADC}_{0-11}
\end{array}\right)=\left[\begin{array}{cccccc}
1 & 1 & 0 & 2 & 0 & 0 \\
1 & 0 & 1 & 0 & 2 & 0 \\
0 & 1 & 1 & 0 & 0 & 2 \\
1 & 1 & 0 & -2 & 0 & 0 \\
1 & 0 & 1 & 0 & -2 & 0 \\
0 & 1 & 1 & 0 & 0 & -2
\end{array}\right]\left(\begin{array}{c}
D_{x x} \\
D_{y y} \\
D_{z z} \\
D_{x y} \\
D_{x z} \\
D_{y z}
\end{array}\right)(1)
$$

The code then inverts the relation of Equation (1) to obtain the diffusion tensor elements, namely $D_{x x}, D_{y y}$, $D_{z z}, D_{x y}, D_{x z}$ and $D_{y z}$ for each pixel in the slice being analysed. From the diffusion tensor the code simply calculates the eigenvalues $\left(\lambda_{1}, \lambda_{2}, \lambda_{3}\right)$ and its corresponding eigenvectors for each tensor belonging to each pixel. The code also calculates FA values at each pixel by the relation:

$$
\mathrm{FA}=\sqrt{\frac{3}{2}} \frac{\sqrt{\left(\lambda_{1}-\lambda\right)^{2}+\left(\lambda_{2}-\lambda\right)^{2}+\left(\lambda_{3}-\lambda\right)^{2}}}{\sqrt{\lambda_{1}^{2}+\lambda_{2}^{2}+\lambda_{3}^{2}}}
$$

where $\lambda$ is the trace of the tensor. The elements of the diffusion tensor were calculated from the diffusionweighted images using a least-square fitting routine, and the tensor was diagonalized to estimate the eigenvalues $\left(\lambda_{1}, \lambda_{2}, \lambda_{3}\right)$ describing the principal diffusivities along its three orthogonal eigenvectors with unitary modulus for each image pixel. These measurements were invariant, meaning independent of the scanner coordinates, and represented the three principal directions of diffusion and their magnitudes within the disc tissue. From the eigenvalues, trace (the mean of the three eigenvalues) and FA values were computed. The FA is a scalar rotationally invariant quantity that describes the degree of anisotropy of diffusion in a tissue. All parameters were calculated pixelwise to map the whole cross-sectional area of the disc. The mean diffusivity of consecutive regions of interest (ROIs) with the same dimensions was plotted against the distance from reference points within the disc and the fractional anisotropy was displayed using both greyand colour-scaled maps. The orientation of the collagen fibres was visualized on the anatomical images from the same slice using a cylinder-shaped surface symbol oriented along the principal eigenvectors (i.e. the eigenvector corresponding to the largest eigenvalue).

\section{Data analysis}

Two observers, an oral and maxillofacial radiologist and high-field MRI expert, were calibrated to review the high-resolution two-dimensional (2D) and 3D images, the orientation of the collagen fibres using the symbols superimposed on the anatomical images, and the grey- and colour-scaled fractional anisotropy maps. The images in each set were displayed on the computer screen and their spatial alignment was confirmed visually. The observers were asked to describe the main orientation of the collagen fibres at each of the selected ROI. They viewed the images twice with a 2-3 week interval between viewing sessions. In case of disagreement, the two observers viewed the images together and reached a consensus. In order to validate the usefulness of DTI to evaluate the structural organization of the TMJ disc, the orientation of the collagen fibres was confirmed via polarized light microscopy on a series of histological slides from the same disc specimens analysed using DTI. For this purpose, tissue slices from each of the three disc bands were cryosectioned at $14 \mu \mathrm{m}$ thickness, mounted on regular glass slides and 
analysed (unstained) under the microscope at $4 \times, 10 \times$ and $20 \times$ magnifications (Nikon E8000; Nikon Instruments Inc., Melville, NY). The images were captured using a charge-coupled device (CCD) camera.

The diffusion properties of the TMJ disc were analysed statistically using ANOVA by comparing the means and standard deviation of the main eigenvalues and fractional anisotropy values on the five samples across the three regions of the disc (Table 1). $P$-values were chosen at 0.05 .

\section{Results}

The 2D axial and coronal GE images of the porcine disc after removal of the temporal bone depict the differences in the fibre orientation between the posterior band (Figure 1c) and intermediate zone (Figure 1b). In the transition area between the posterior band and the intermediate zone, a combination of long axes and cross-sections of fibres are visualized (Figure 1d). The findings from the axial and coronal gradient echo images were confirmed using polarized light microscopy (Figure 1e-g).

The 3D views of the porcine disc attached to the condyle were obtained by applying a wedge function to segment out a portion of the image volume allowing the simultaneous visualization of the internal structure of the TMJ disc in the coronal, sagittal and axial planes (Figure 2a-d). In these images, the fibres in the anterior and posterior bands of the disc are orientated mediolaterally (white arrows in Figure 2a,c) whereas those from the intermediate zone run in an anteriorposterior direction (black arrows in Figure 2b,d).

As an example of the DTI data obtained as part of this study, a coronal anatomical image of the intact disc is presented (Figure 3a) for which its corresponding FA (Figure 3b) and trace maps (Figure 3c) were computed

Table 1 Mean diffusivities along the (a) sagittal and (b) coronal planes. The mean diffusivity is lower in the intermediate zone in the sagittal plane and in the central area in the coronal plane. (c) Statistical result (mean \pm standard deviation) of the measurements and estimates of the diffusion eigenvalues $\left(\lambda_{1}, \lambda_{2}, \lambda_{3} \times 10^{-3} \mathrm{~mm}^{2} \mathrm{~s}^{-1}\right)$ and the fractional anisotropy (FA) index for the three regions of interest (outlined in Figure 3a) across the disc of the normal porcine temporomandibular joint, $n=5$

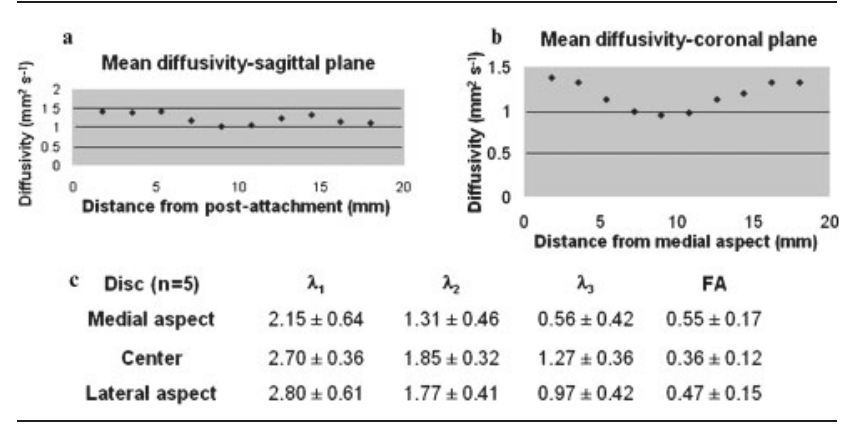

from the DTI data acquired from the same slice. The measurements of the diffusion properties on the selected ROIs marked on the anatomical image in Figure $3 \mathrm{a}$ were analysed statistically using ANOVA and the results are presented in Table 1c. The collagen fibre orientation in $3 \mathrm{D}$ is displayed by representing the principal eigenvectors as small red lines overlaid on each voxel of the anatomical image (Figure 3d). The regional variations in the eigenvector directions are in agreement with the expected fibre orientation confirming the ability of DTI measurements to sensitively detect the fibres' microstructural organization. All five samples showed comparable behaviour of the mean diffusivity, FA index and the eigenvector corresponding to the largest eigenvalues (Table 1c).

In the sagittal plane, the mean diffusivity was higher in the posterior $\left(1.28 \pm 0.10 \times 10^{-3} \mathrm{~mm}^{2} \mathrm{~s}^{-1}\right)$ and anterior $\left(1.27 \pm 0.08 \times 10^{-3} \mathrm{~mm}^{2} \mathrm{~s}^{-1}\right)$ bands of the disc compared with the intermediate zone $(0.96 \pm 0.01 \times$ $10^{-3} \mathrm{~mm}^{2} \mathrm{~s}^{-1}$ ) (Table 1a). These values corresponded to the fractional anisotropy index, which was lower in the intermediate zone compared with the anterior and posterior bands (data not shown).

In the coronal plane, the mean diffusivity was higher in the medial $\left(1.42 \pm 0.01 \times 10^{-3} \mathrm{~mm}^{2} \mathrm{~s}^{-1}\right)$ and lateral $\left(1.21 \pm 0.12 \times 10^{-3} \mathrm{~mm}^{2} \mathrm{~s}^{-1}\right)$ aspects of the posterior band than at the centre $\left(1.09 \pm 0.08 \times 10^{-3} \mathrm{~mm}^{2} \mathrm{~s}^{-1}\right)$ (Table 1b). In this plane, the fractional anisotropy index was lower in the central aspect of the disc followed by the medial and the lateral regions (Figure 3c).

The eigenvector projection map showed regional variations depending on the location and the plane. These variations support the specialization of the tissue according to the function and the properties of each region. For example, in the sagittal plane the vectors in the posterior and anterior bands presented an out-ofplane orientation whereas those in the intermediate zone had an in-plane alignment. In contrast, Figure $3 \mathrm{~d}$ illustrates the in-plane orientation of the majority of the eigenvectors in a coronal view at the level of the posterior band.

\section{Discussion}

There are a wide variety of techniques available for imaging the TMJ. The reason for such a large number of techniques include the great biological variation of the bony structures, the fact that techniques that reproduce bone structures well are frequently poorly suited for imaging soft tissues and vice versa. In addition, the 3D alignment of the condyle often leads to distortion of the condyle-fossa relationship unless the angle of radiation is individualized. Another limitation associated with the most common imaging modalities, e.g. panoramic radiography, tomography and $\mathrm{CT}$, is the use of ionizing radiation. Moreover, imaging procedures are almost never useful as primary 


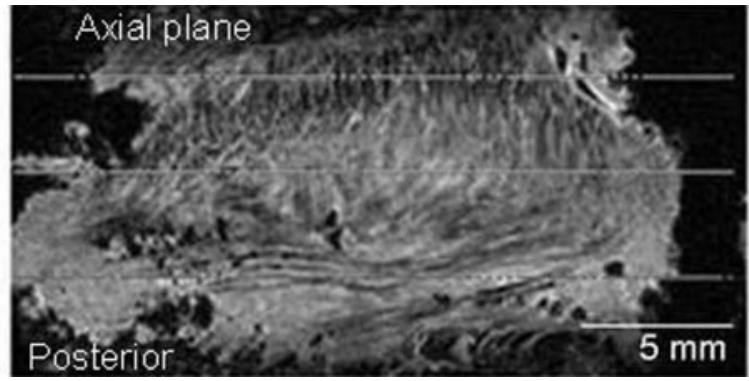

a

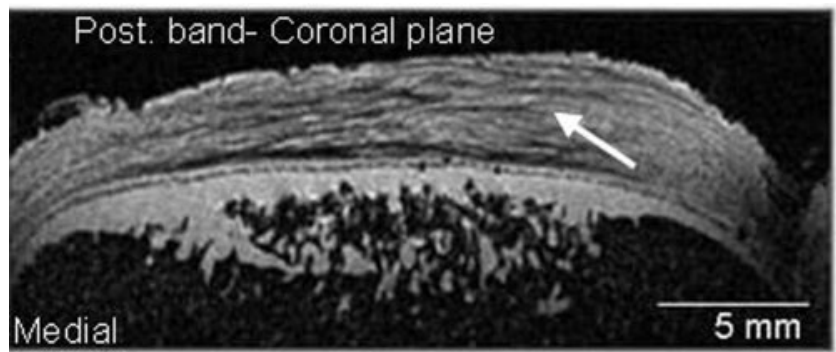

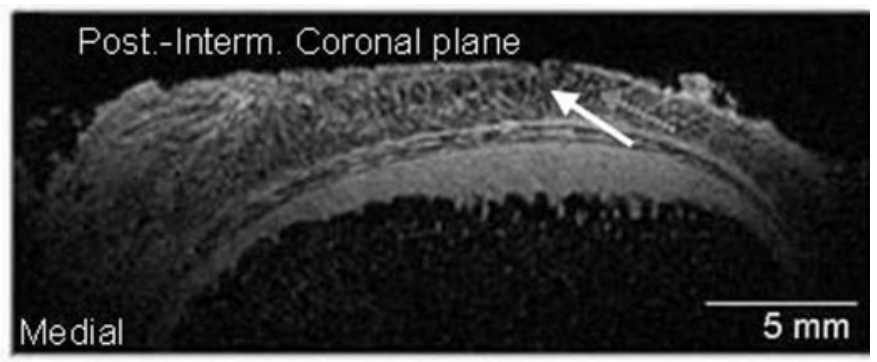

b

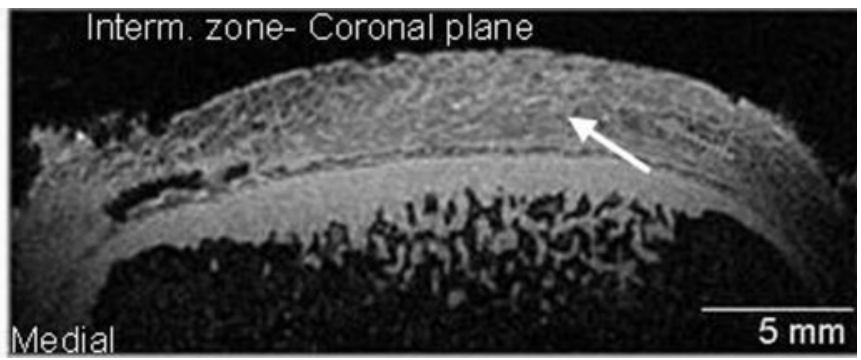

d

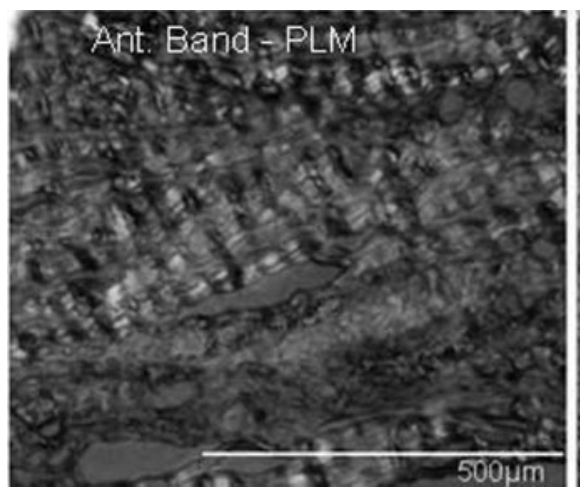

e

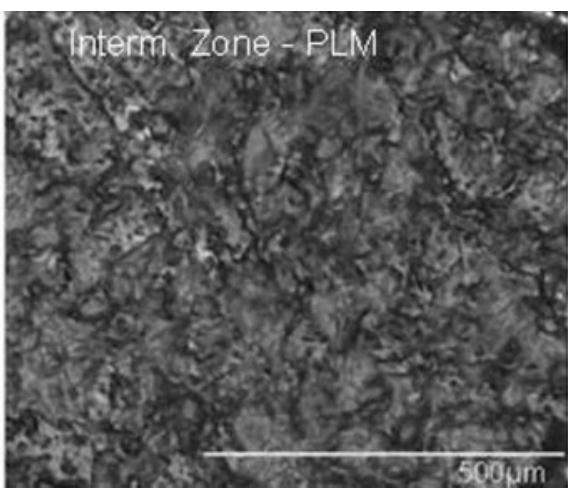

f

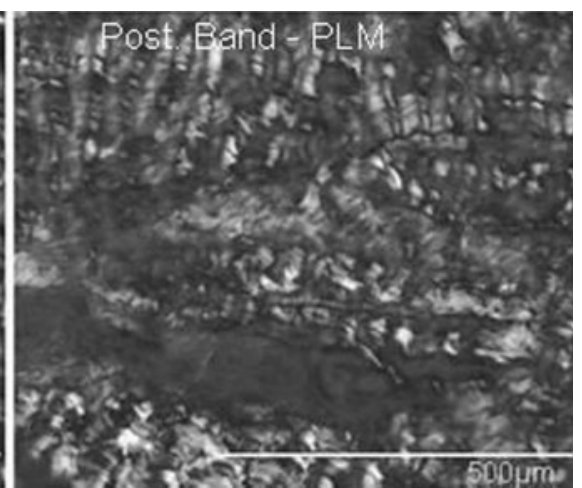

g

Figure 1 Gradient-echo MRIs of the porcine disc attached to the condyle in the (a) axial and (b-d) coronal planes. (a) The grey lines indicate the level of the corresponding coronal images. Note the difference in fibre orientation between (c) the posterior band, (b) the transition zone between the posterior band and the intermediate zone and (d) intermediate zone (arrows). (c) In the posterior band, the long axis of the fibres can be visualized running along the mediolateral direction (white arrow). In contrast, the fibres in the intermediate zone run in the anteroposterior direction; therefore, (b) the MRI in the coronal plane shows a cross-section of them (arrow). (e-g) Polarized light microscopy images of the temporomandibular disc showing the fibre orientation at the (e) anterior, (f) intermediate and (g) posterior regions. The tissue slides were sectioned parallel to the coronal plane of the joint. The striped pattern indicates the crimping and banding of the fibres. In the anterior and posterior bands the fibres run parallel from left to right, indicating mediolateral orientation of their long axis, whereas in the intermediate zone only the cross-section of the fibre bundles is visualized

tools for diagnosing functional disturbance. In general, the imaging modalities provide information about the form and position of structures. The occurrence of symptoms in TMD depends primarily on function and the capacity for adaptation. In this regard, one must give special attention to the functional adaptability of the non-bony structural components of the joint. With the exception of MRI, imaging procedures cannot reveal evidence of adaptation in the bilaminar zone, the fibrocartilaginous articular surfaces or the disc. ${ }^{30}$

Emerging MRI technologies are now allowing investigators to move beyond the current capabilities of anatomic definition and determine hydration, collagen and proteoglycan content of tissues. DTI is one of these emerging tools, that was developed originally to provide information about axonal integrity and the course of white matter fibre tracts in the central nervous system. ${ }^{31}$ Briefly, this technique exploits the perpetual and random motion of water molecules in tissues and provides an additional degree of contrast that could help with pathological characterization of the tissue. In the absence of boundaries, water diffusion is isotropic and characterized by a single parameter, generally referred to as ADC. However, in organized or semi-organized structures, such as axons or collagen fibres of the TMJ disc, water diffuses in preferential directions. Water molecules tend to diffuse faster along the fibres compared with 


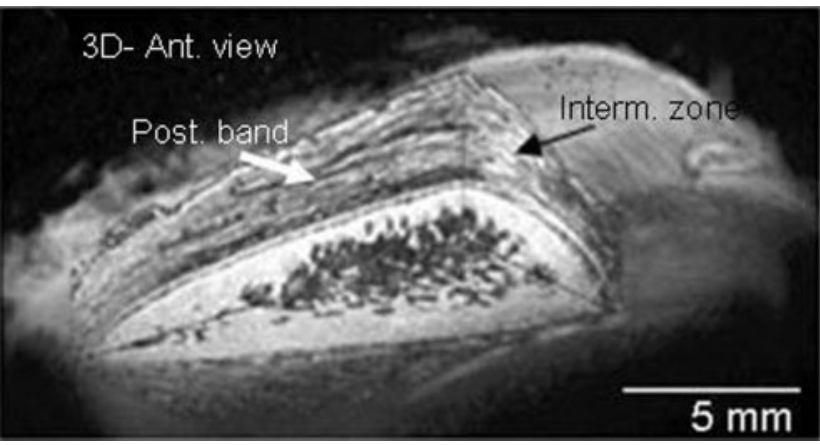

a

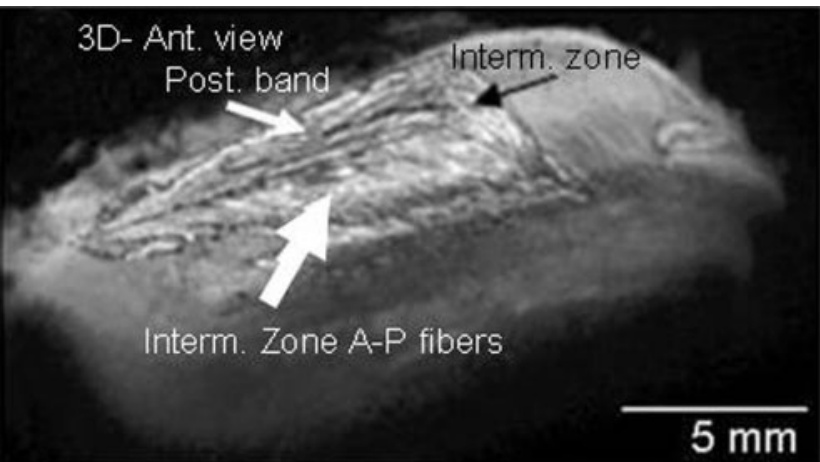

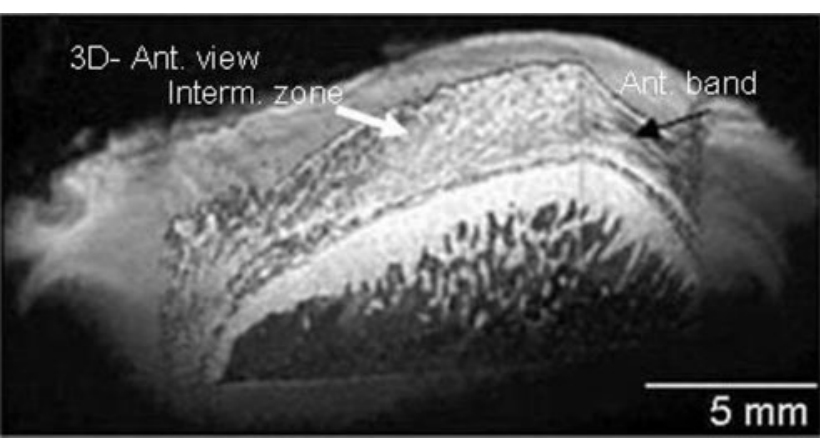

b

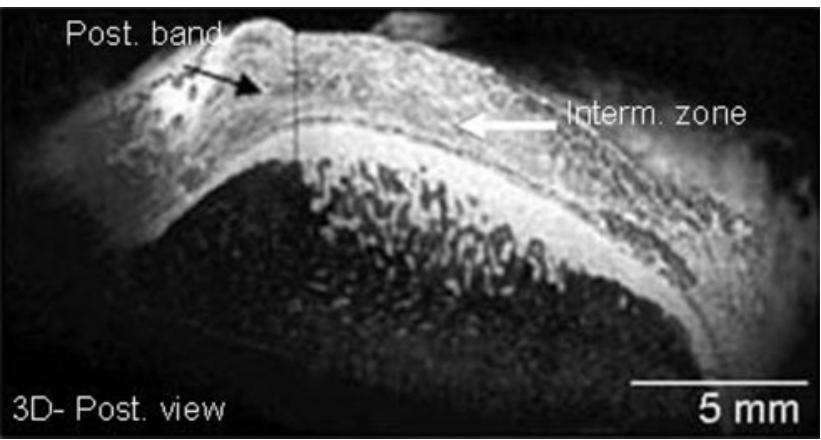

d

Figure 2 Three-dimensional visualization of the porcine disc attached to the condyle. The gradient-echo images are shown in a wedged format to simultaneously display the coronal, sagittal and axial cross-sectional cuts from the disc. (a-c) Depict the inherent organization of the fibres in an anterior view by segmenting out the anterolateral portion of the volume data. (d) Represents a posterior view of the disc in which the posterolateral portion of the volume data has been suppressed. In (a,c) the black arrows show the long axis of the fibres in the intermediate zone, whereas the white arrows display the fibres in the posterior band. The thick white arrow in (c) is showing the anteroposterior direction of the intermediate zone fibres viewed in the axial plane. In (b,d) the black arrows represent the long axis of the fibres in the anterior and posterior bands, respectively, whereas the white arrows show the cross-section of the fibres in the intermediate zone in an (b) anterior and (d) posterior view

transverse directions. This directionality is known as diffusion anisotropy. The degree of anisotropy reflects fibre integrity that is not evident on conventional MRI.

In this study, we tested for the first time the feasibility of performing DTI measurements to quantitatively evaluate the microstructural organization of these fibres and the disc's water diffusion properties. The data collectively indicate that the TMJ disc presents distinct regional variations in fibre organization. These results were confirmed by using polarized light microscopy as the gold standard. As expected, the diffusion of water is anisotropic, but the posterior and anterior bands exhibit greater anisotropy and principal diffusivity than the intermediate zone.

One of the commonly used scalar invariant quantities to characterize diffusion in tissues is FA. ${ }^{32,33}$ FA receives a value closer to 0 for fully isotropic diffusion as in liquid, and to 1 for complete anisotropy. Variations of the mean diffusivity and FA were observed along the sagittal and coronal planes confirming the heterogeneous nature of this fibrocartilaginous TMJ disc tissue. FA maps appear to be the most promising information for characterizing the diffusion properties of the TMJ disc.
The MRI and DTI results confirm the well established evidence about regional variations and specializations observed within the TMJ disc. The DTI results offer the unique advantage of providing not only qualitative but also quantitative microstructural and property information in a non-invasive manner. This first study to adapt DTI to the evaluation of the TMJ disc demonstrates the capabilities of this technique for determining the diffusion properties of the TMJ disc and microstructural analysis of the collagen fibre distribution and orientation.

We applied high-resolution MRI modalities and demonstrated their merits in characterizing the morphology, the microstructure and the diffusion properties of the TMJ disc. We showed that the GE-based anatomical imaging is capable of producing sensitive and specific data to depict the morphology and orientation of the disc fibres which was further supported by our polarized light microscopy observations.

To summarize the significance of this study, it is important to keep in mind that previous authors have reported that the regular pattern of extracellular matrix organization is disrupted as a consequence of disease or 


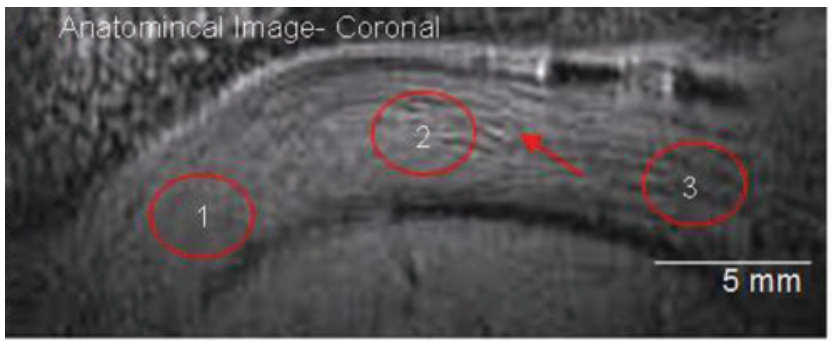

a

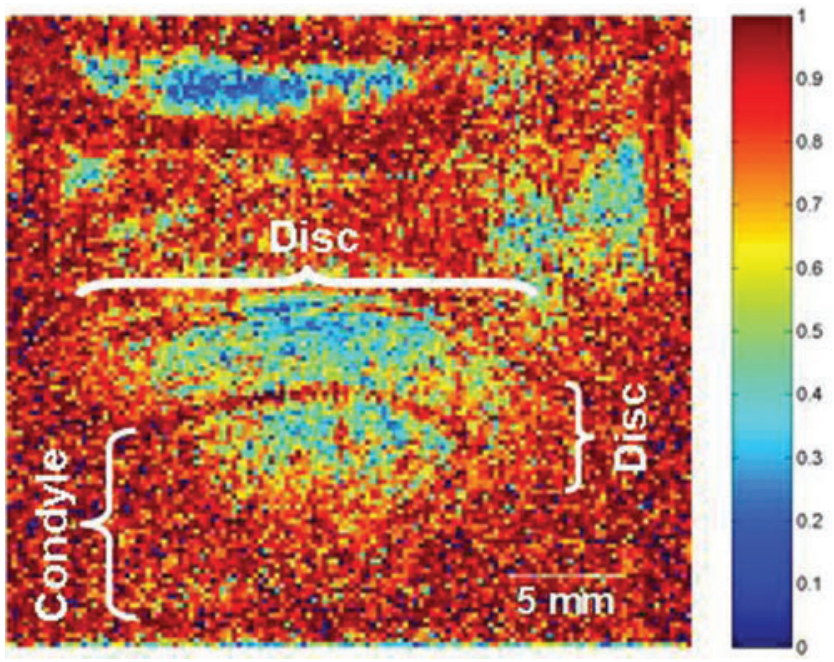

c

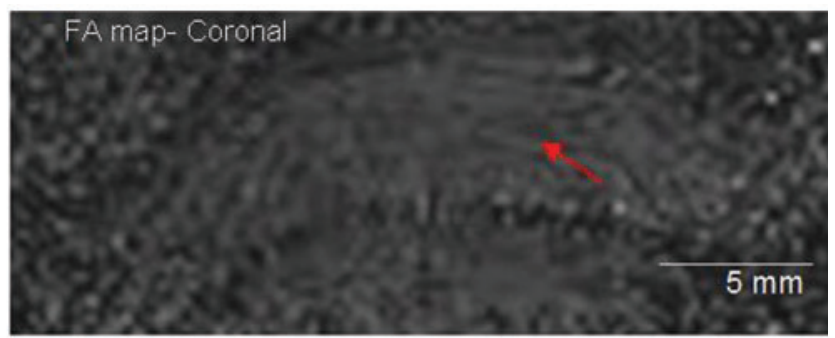

b

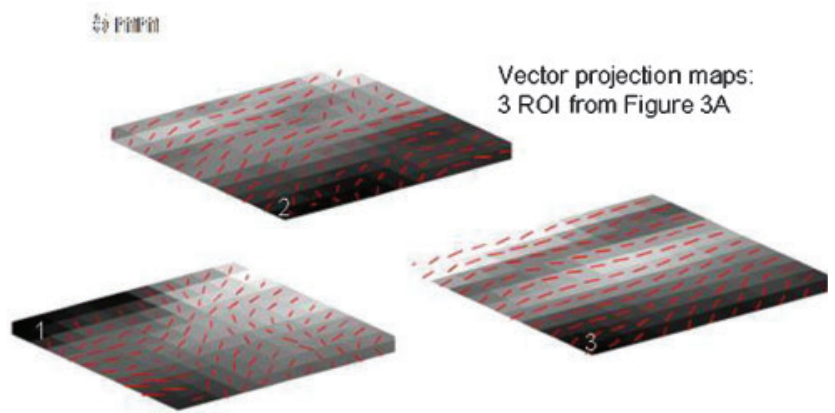

d

Figure 3 (a) Anatomical image and (b) corresponding fractional anisotropy map of the intact joint in the coronal plane. Note the parallel orientation of collagen fibres in the disc (red arrow). (c) Colour-scaled trace map displays differences in the degree of anisotropy across the tissue from low $\left(0\right.$, blue) to high $\left(1\right.$, red). (d) Vector projection maps of eigenvalues $\left(\lambda_{1}, \lambda_{2}, \lambda_{3}\right)$ representing the principal diffusivities of water in the three regions of interest (ROIs) outlined in red in (a). The vectors were estimated from diffusion tensor imaging measurements

disc displacement. 6,8 For example, an early and key event in degenerative joint disease is loss of the integrity of the collagenous matrix of articulating structures. These early degenerative changes characteristically occur long before any disruption is detected using current imaging modalities. Given that the mechanical properties of the TMJ disc are largely dependent on the collagen fibre organization, proteoglycan composition and the interaction of these components with the tissue fluid, and that the initial effect of collagen degradation in articular tissues is an increase in their water content, it is reasonable to believe that early degenerative changes within the disc would be reflected in the measurement of its diffusion properties. ${ }^{34-36}$ Our quantitative results encourage the use of DTI for comparison of similar measurements obtained from the TMJ discs of experimentally induced disease or inflammation in animal models such as the miniature pig model that correlates degenerative joint disease following intraoral splint wear described by Sindelar and coworkers. ${ }^{37,38}$

In regards to the use of DTI, a much higher magnetic field strength than is currently approved for clinical studies was used. The use of this high field strength provided increased signal-to-noise ratio and resolution necessary in the case of this study; however, it leaves the question open as to whether this technique would be feasible for human use. Although the results from the present investigation suggest that DTI could be a critical tool in the detection of early pathologies of the TMJ disc, additional work needs to be performed to optimize DTI protocols and start testing this modality at lower field strengths that more closely resemble the current clinical imaging situation.

\section{Acknowledgments}

This work was supported by the National Institute for Dental and Craniofacial Research (Grant \# T32 DE07294) and the UMKC Center for Research on Interfacial Structure and Properties (UMKC-CRISP). The authors are grateful to Dr James Cook from the Comparative Orthopedic Laboratory and the Swine Resource Center at the University of MissouriColumbia for providing the porcine specimens used in this study; Dr Terence McIff and Mr Richard Lark from the Orthopedic Surgery Laboratory at the Kansas University Medical Center for assisting with the specimen dissection; and, Mrs Rebecca Chambers from the Hoglund Brain Imaging Center at the Kansas University Medical Center for her support with the 3D data processing. 


\section{References}

1. Nickel JC, Iwasaki LR, Feely DE, Stormberg KD, Beatty MW. The effect of disc thickness and trauma on disc surface friction in the porcine temporomandibular joint. Arch Oral Biol 2001; 46: $155-162$.

2. Nickel JC, McLachlan KR. In vitro measurement of the stressdistribution properties of the pig temporomandibular joint disc. Arch Oral Biol 1994; 39: 439-448.

3. Mills DK, Fiandaca DJ, Scapino RP. Morphologic, microscopic, and immunohistochemical investigations into the function of the primate TMJ disc. J Orofac Pain 1994; 8: 136-154.

4. Detamore MS, Orfanos JG, Almarza AJ, French MM, Wong ME, Athanasiou KA. Quantitative analysis and comparative regional investigation of the extracellular matrix of the porcine temporomandibular joint disc. Matrix Biol 2005; 24: 45-57.

5. Takisawa A, Ihara K, Jinguji Y. Fibro-architectonics of human temporomandibular joint. Okajimas Folia Anat Jpn 1982; 59: 141-166.

6. Scapino RP. Histopathology associated with malposition of the human temporomandibular joint disc. Oral Surg Oral Med Oral Pathol 1983; 55: 382-397.

7. Moffet BC. Histologic aspects of temporomandibular joint derangements. In: Moffet BC and Westesson PL (eds). Diagnosis of internal derangements of the temporomandibular joint. Vol. 1. Double-Contrast Arthrography and Clinical Correlation. Seattle, WA: University of Washington Continuing Education, 1984, pp 47-49

8. Tallents RH, Macher DJ, Rivoli P, Puzas JE, Scapino RP, Katzberg RW. Animal model for disk displacement. $J$ Craniomandib Disord 1990; 4: 233-240.

9. Katzberg RW, Schenck J, Roberts D, Tallents RH, Manzione JV Hart HR, et al. Magnetic resonance imaging of the temporomandibular joint meniscus. Oral Surg Oral Med Oral Pathol 1985; 59: 332-335.

10. Tasaki MM, Westesson PL. Temporomandibular joint: diagnostic accuracy with sagittal and coronal MR imaging. Radiology 1993; 186: 723-729.

11. Tasaki MM, Westesson PL, Kurita K, Mohl N. Magnetic resonance imaging of the temporomandibular joint. Value of axial images. Oral Surg Oral Med Oral Pathol 1993; 75: 528-531.

12. Westesson PL. Reliability and validity of imaging diagnosis of temporomandibular joint disorder. Adv Dent Res 1993; 7: 137-151.

13. Matsuda S, Yoshimura Y, Lin Y. Magnetic resonance imaging assessment of the temporomandibular joint in disk displacement. Int J Oral Maxillofac Surg 1994; 23: 266-270.

14. Bermejo A, Gonzalez O, Gonzalez JM. The pig as an animal model for experimentation on the temporomandibular articular complex. Oral Surg Oral Med Oral Pathol 1993; 75: 18-23.

15. Dannhauer KH, Grunder W, Rumpel H, Gersonde K. [NMRmicroscopic studies of the condylar cartilage of the temporomandibular joint]. Fortschr Kieferorthop 1990; 51: 248-251 (in German).

16. Dannhauer KH, Sergl HG, Grunder W. [The demonstration of cellular reactions in the temporomandibular condylar cartilage by NMR microscopy. The basic research for high-resolution NMR tomography of the temporomandibular joint]. Fortschr Kieferorthop 1992; 53: 49-52 (in German).

17. Pierpaoli C, Basser PJ. Toward a quantitative assessment of diffusion anisotropy. Magn Reson Med 1996; 36: 893-906.

18. Virta A, Barnett A, Pierpaoli C. Visualizing and characterizing white matter fiber structure and architecture in the human pyramidal tract using diffusion tensor MRI. Magn Reson Imaging 1999; 17: 1121-1133.

19. Holmes AA, Scollan DF, Winslow RL. Direct histological validation of diffusion tensor MRI in formaldehyde-fixed myocardium. Magn Reson Med 2000; 44: 157-161.

20. Elshafiey I, Bilgen M, Narayana PA. In vivo diffusion tensor imaging of rat spinal cord at 7T. Magn Reson Imaging 2002; 20: 243-247.

21. Lythgoe MF, Busza AL, Calamante F, Sotak CH, King MD, Bingham AC, et al. Effects of diffusion anisotropy on lesion delineation in a rat model of cerebral ischemia. Magn Reson Med 1997; 38: 662-628.

22. van Gelderen P, de Vleeschouwer MH, DesPres D, Pekar J, van Zijl PC, Moonen CT. Water diffusion and acute stroke. Magn Reson Med 1994; 31: 154-163.

23. Werring DJ, Clark CA, Barker GJ, Thompson AJ, Miller DH. Diffusion tensor imaging of lesions and normal-appearing white matter in multiple sclerosis. Neurology 1999; 52: 1626-1632.

24. Le Bihan D. Molecular diffusion nuclear magnetic resonance imaging. Magn Reson $Q$ 1991; 7: 1-30.

25. Basser PJ, Mattiello J, LeBihan D. MR diffusion tensor spectroscopy and imaging. Biophys $J$ 1994; 66: 259-267.

26. Basser PJ. Inferring microstructural features and the physiological state of tissues from diffusion-weighted images. NMR Biomed 1995; 8: 333-344.

27. Pierpaoli C, Jezzard P, Basser PJ, Barnett A, Di Chiro G. Diffusion tensor MR imaging of the human brain. Radiology 1996; 201: 637-648.

28. Basser PJ, Pierpaoli C. Microstructural and physiological features of tissues elucidated by quantitative-diffusion-tensor MRI. $J$ Magn Reson B 1996; 111: 209-219.

29. Bilgen M. Inductively-overcoupled coil design for high resolution magnetic resonance imaging. Biomed Eng Online 2006; 5: 3.

30. Bumann A. TMJ disorders and orofacial pain: the role of dentistry in a multidisciplinary diagnostic approach (color atlas of dental medicine) (1st edn). New York: Thieme, 2002.

31. Le Bihan D, Mangin JF, Poupon C, Clark CA, Pappata S, Molko $\mathrm{N}$, et al. Diffusion tensor imaging: concepts and applications. $J$ Magn Reson Imaging 2001; 13: 534-546.

32. Bilgen M, Elshafiey I, Narayana PA. Mohr diagram representation of anisotropic diffusion tensor in MRI. Magn Reson Med 2002; 47: 823-827.

33. Bilgen M, Narayana PA. Mohr diagram interpretation of anisotropic diffusion indices in MRI. Magn Reson Imaging 2003; 21: 567-572.

34. Torzilli PA, Grigiene R, Borrelli J Jr., Helfet DL. Effect of impact load on articular cartilage: cell metabolism and viability, and matrix water content. J Biomech Eng 1999; 121: 433-441.

35. Herwig J, Egner E, Buddecke E. Chemical changes of human knee joint menisci in various stages of degeneration. Ann Rheum Dis 1984; 43: 635-640.

36. Altman RD, Tenenbaum J, Latta L, Riskin W, Blanco LN, Howell DS. Biomechanical and biochemical properties of dog cartilage in experimentally induced osteoarthritis. Ann Rheum Dis 1984; 43: 83-90.

37. Sindelar BJ, Evanko SP, Alonzo T, Herring SW, Wight T. Effects of intraoral splint wear on proteoglycans in the temporomandibular joint disc. Arch Biochem Biophys 2000; 379: 64-70.

38. Sindelar BJ, Edwards S, Herring SW. Morphologic changes in the TMJ following splint wear. Anat Rec 2002; 266: 167-176. 